\title{
The status and preliminary results of the LHC forward experiment: LHCf
}

\author{
H. MENJO ${ }^{* a}$, O. Adriani ${ }^{a b}$, L. Bonechi ${ }^{a}$, M. Bongi ${ }^{a}$, G. Castellini ${ }^{a c}$, R. D'Alessandro ${ }^{a b}$, \\ A. Faus ${ }^{d}$, K. Fukatsu ${ }^{e}$, M. Haguenauer ${ }^{f}$, Y. Itow $^{e}$, K. Kasahara ${ }^{g}$, K. Kawade ${ }^{e}$, \\ D. Macina ${ }^{h}$, T. Mase $^{e}$, K. Masuda ${ }^{e}$, Y. Matsubara ${ }^{e}$, G. Mitsuka ${ }^{e}$, Y. Muraki ${ }^{i}$, M. Nakai ${ }^{g}$, \\ K. Noda ${ }^{e}$, P. Papini ${ }^{a}$, A-L. Perrot ${ }^{h}$, S. Ricciarini ${ }^{a}$, T. Sako ${ }^{e}$, G. Sinatra ${ }^{l}$, K. Suzuki ${ }^{e}$, \\ T. Suzuki ${ }^{g}$, Y. Shimizu ${ }^{g}, \mathbf{K}$. Taki $^{e}$, T. Tamura $^{j}$, S. Torii $^{g}, \mathbf{A}$. Tricomi $^{k l}$, J. Velasco $^{d}$, \\ W. C. Turner ${ }^{m}$ and K. Yoshida ${ }^{n}$ \\ ${ }^{a}$ INFN Sezione di Firenze, Italy \\ ${ }^{b}$ Università degli Studi di Firenze, Florence, Italy \\ ${ }^{c}$ IFAC CNR, Florence, Italy \\ ${ }^{d}$ IFIC, Universitat de València, Valencia, Spain \\ ${ }^{e}$ Solar-Terrestrial Environment Laboratory, Nagoya University, Nagoya, Japan \\ ${ }^{f}$ Ecole-Polytechnique, Palaiseau Cedex, France \\ ${ }^{g}$ RISE, Waseda University, Tokyo, Japan \\ ${ }^{h}$ CERN, Genève, Switzerland \\ ${ }^{i}$ Department of Physics, Konan University, Koube, Japan \\ ${ }^{j}$ Institute of Physics, Kanagawa University, Yokohama, Japan \\ ${ }^{k}$ Università degli Studi di Catania, Catania, Italy \\ ${ }^{l}$ INFN Sezione di Catania, Catania, Italy \\ ${ }^{m}$ Accelerator and Fusion Research Division, LBNL, Berkeley, USA \\ ${ }^{n}$ Faculty of System Engineering, Shibaura Institute of Technology, Saitama, Japan \\ E-mail: menjo@fi.infn.it
}

The LHCf is one of the LHC forward experiments and the aim is to measure energy and transverse momentum spectra of gamma-rays and neutral hadrons emitted in the very forward region of LHC. In 2009, LHC started the operation and LHCf has taken data at $\sqrt{s}=900 \mathrm{GeV}$. The measured spectra of gamma-ray like events and hadron like events are in good agreement with the Monte-Carlo simulation results generated by the QGSJET2 model within error. In 2010, LHC are delivering $p p$ collisions at $\sqrt{s}=7 \mathrm{TeV}$ and LHCf are taking data with very low beam-gas background level of less than $1 \%$.

XVIII International Workshop on Deep-Inelastic Scattering and Related Subjects, DIS 2010 April 19-23, 2010

Firenze, Italy

\footnotetext{
*Speaker.
} 


\section{Introduction}

The Monte-Carlo(MC) simulation is a part of essential tools for measurements of high energy cosmic rays. However hadron interaction models used in air shower simulations have an uncertainty due to the lack of experimental data in the energy range over $10^{15} \mathrm{eV}$. The Large Hadron Collider (LHC), which is the largest and the most powerful hadron collider, started the operation in 2009. The design collision energy is $\sqrt{s}=14 \mathrm{TeV}$, which corresponds to $10^{17} \mathrm{eV}$ in the laboratory system. The LHCf experiment is one of the LHC forward experiments. The aim is to measure the energy and transverse momentum spectra of gamma-rays and neutral hadrons emitted in the very forward region of LHC and to provide critical data for calibration of hadron interaction models.

\section{LHCf Experiment}

The LHCf apparatus is composed of independent detectors (Arm\#1 and Arm\#2) with similar design but with slightly different configuration for background rejection and redundancy. Each detector have two sampling and imaging calorimeters with the transverse cross section of $20 \times 20 \mathrm{~mm}$ and $40 \times 40 \mathrm{~mm}$ in the Arm\#1 detector and $25 \times 25 \mathrm{~mm}$ and $32 \times 32 \mathrm{~mm}$ in the Arm\#2 detector. Figure 1 shows the schematic view of the Arm\#1 and Arm\#2 detectors. Each calorimeter is composed of 22 tungsten plates of $7 \mathrm{~mm}$ thickness (2r.1.), 16 plastic scintillators and 4 position sensitive layers of X-Y SciFi hodoscopes and X-Y silicon strip detectors for Arm\#1 and Arm\#2, respectively. The plastic scintillators are inserted by 2 or 4 r.l. step for shower sampling and The position sensitive layers are inserted at 6,10,30 and 42 r.l. for the Arm1 calorimeters and at 6, 12, 30 and 42 r.l. for the Arm 2 calorimeters to measure the shower position and identify the multiple showers in one calorimeter. The total thickness of each calorimeter is 44 r.l. and 1.7 interaction lengths. The calorimeters of each detector together with PMT's and front-end circuits are packed in the aluminum box of $92 \mathrm{~mm}^{w} \times 280 \mathrm{~mm}^{l} \times 620 \mathrm{~mm}^{h}$. The energy resolution is expected to be $<5 \%$ and about $30 \%$ for gamma-rays with more than $100 \mathrm{GeV}$ and hadrons, respectively. The position resolution for gamma-ray showers is expected to be better than $0.2 \mathrm{~mm}$. These detector performances have been confirmed at the beam tests done in 2006 and 2007 at CERN SPS [1].
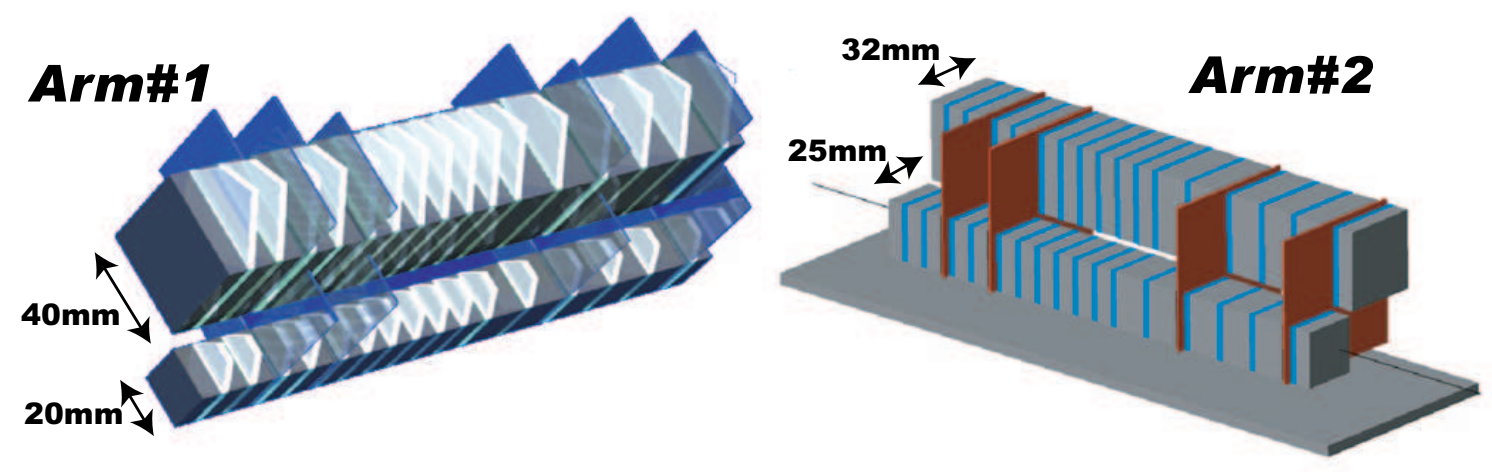

Figure 1: Schematic view of the Arm\#1 and Arm\#2 detectors. 
At $+/-140 \mathrm{~m}$ from an LHC interaction point, IP1, zero degree neutral absorbers (TAN's) have been installed for preventing quenching of downstream superconducting magnets by neutral particles produced in $p p$ collisions. Inside the TAN, the beam pipe makes a transition from a single common beam pipe facing IP1 to two separate beam pipes joining to the arcs of LHC (Y-chamber). In the crotch of the Y-chamber, there is an instrumentation slot with dimensions $96 \mathrm{~mm}^{w} \times 1000 \mathrm{~mm}^{l} \times 607 \mathrm{~mm}^{h}$ for detectors of beam monitors and forward physics experiments. The LHCf detectors have been inserted in the slots of the both TAN's. The instrumentation slot allow to measure neutral particles produced at the interaction point at zero degree of collisions but not charged particles because they are swept away by the magnetic field of the beam separation dipoles located between IP1 and the TAN. The aperture is limited by the size of the TAN instrumentation slot and the aperture of the beam pipes located between IP1 and the TAN. The pseudo-rapidity coverage of the LHCf detectors is $\eta>8.7$ and $\eta>8.4$ with zero and $140 \mu \mathrm{rad}$ beam crossing angles, respectively.

\section{Results at $900 \mathrm{GeV}$ in 2009}

LHC have delivered proton beams with $450 \mathrm{GeV}$ beam energy in December 2009 and produced about $5 \times 10^{5}$ pp collisions without beam crossing angle at IP1. We have operated for 2.6 hours and 27.7 hours for detector commissioning and physics, respectively. We have collected about 2,800 and 3,600 shower events in total of operations in 2009 by Arm1 and Arm2 detectors, respectively. About $10 \%$ of the events are background events due to collisions between beams and residual gas in the beam pipes (beam-gas background). The background can be estimated by using events measured with non-colliding beams at IP1.

Electromagnetic showers induced by gamma-rays are discriminated from hadronic showers by using the longitudinal shower development measured by the 16 scintillator layers. To parameterize the transition shape of a shower event by event, we defined L90\%, which is the longitudinal position containing $90 \%$ of the sum of the shower particles. Figure 2 shows the L90\% distribution of data in 2009 measured by the smaller calorimeter of the Arm\#1 detector. Electromagnetic showers develop

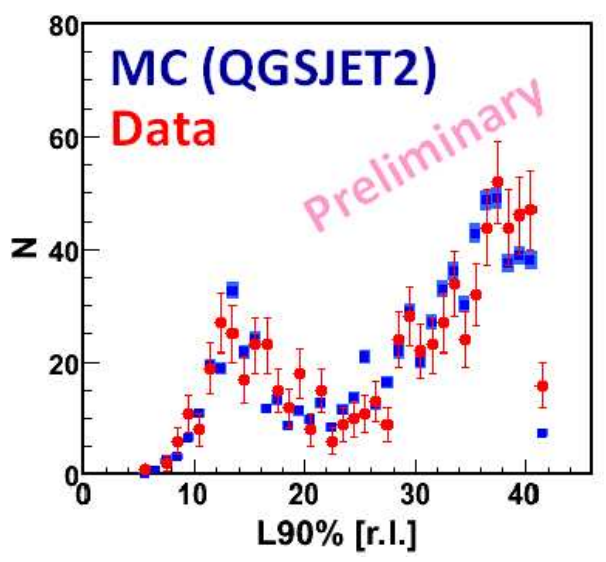

Figure 2: L90\% distributions at the Arm\#1 smaller calorimeter. The red and blue points indicate results of data and MC generated by the QGSJET2 model, respectively. 
more rapidly than hadronic showers and make the peak of L90\% distribtion at around 12 r.l.. The L90\% distribution expected by MC simulation data with $10^{7}$ collisions generated by the QGSJET2 model [2] is also shown as blue dots in fig.2. The L90\% distribution of data is in good agreement with that of MC. We set the criteria of $\mathrm{L} 90 \%$ to discriminate between gamma-ray like events and hadron like events as a function slightly depending on total energy deposit $(d E)$ in the calorimeter, $16+0.0002 \times d E$ r.1.. $d E$ is total energy deposit normalized by MIP and it is about 4000 when a gamma-ray with $100 \mathrm{GeV}$ hits the calorimeter

Figure 3 shows the spectra of gamma-ray and hadron like events measured by the Arm\#1 and Arm\#2 detectors after the subtraction of beam-gas background estimated by using non-colliding beams at IP1. The blue dots in fig 3 shows MC results normalized by entries of gamma-ray like events. The measured spectra are in good agreement with MC results within statistical error. In the spectra for hadron like events, for the moment, the gamma-ray equivalent energy for are used instead of reconstructed hadron energy because still the detector response for hadrons is under study by using beam test data with $150 \mathrm{GeV}$ and $350 \mathrm{GeV}$ protons. The systematic errors are also under study. For the moment, we set $+11 \% /-5 \%$ error of energy scale.
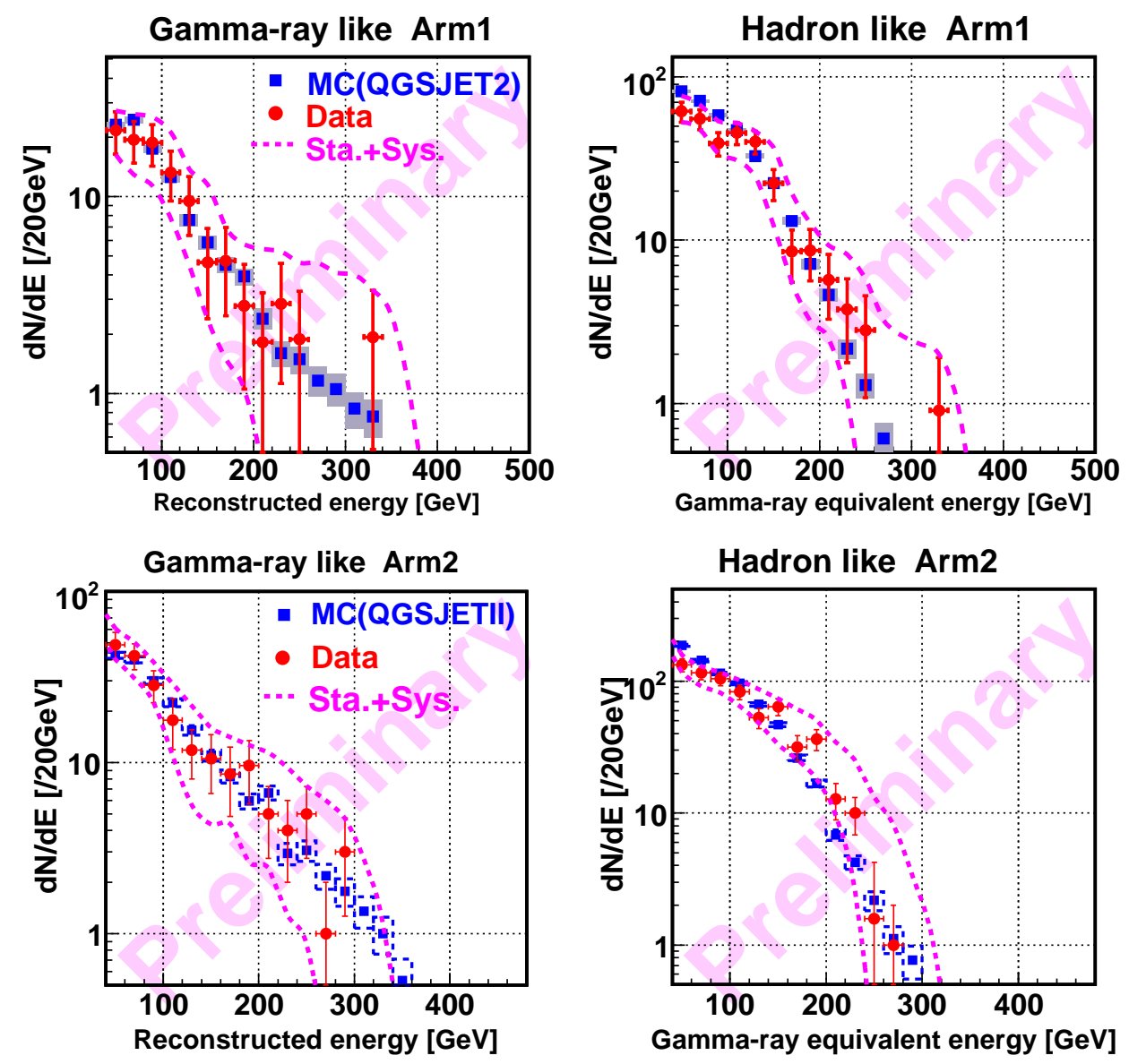

Figure 3: Energy spectra of gamma-ray like events and hadron like events measured by the Arm\#1 and Arm\#2 detectors. The red and blue dots show data and MC generated with the QGSJET2 model. The MC spectra are normalized by entries of gamma-ray like events in each detector. 


\section{Operation in 2010}

On March 30, 2010, LHC had the first collision with 3.5TeV proton beams. The collision energy of $\sqrt{s}=7 \mathrm{TeV}$ corresponds to $2.6 \times 10^{16} \mathrm{eV}$ in the laboratory system. LHCf has collected about $7 \times 10^{4}$ shower events for 3 hours at the day. Figure 4 shows the spectra of gamma-ray like and hadron like events measured by the Arm\#2 smaller calorimeter. The beam-gas background level at $7 \mathrm{TeV}$ collisions are less than $1 \%$, which is much better than that at $900 \mathrm{GeV}$ collisions in 2009. After the day, LHC is delivering collisions at $7 \mathrm{TeV}$ with increasing the luminosity step by step and LHCf are taking data. The LHCf detectors will be removed from the LHC tunnel when the luminosity reaches $10^{30} \mathrm{~cm}^{-2} \mathrm{~s}^{-1}$ or the integral luminosity reaches $2 \mathrm{nb}^{-1}$. The LHCf detectors will be re-installed to the LHC tunnel in 2013 for operation at the LHC design energy of $\sqrt{s}=14 \mathrm{TeV}$.
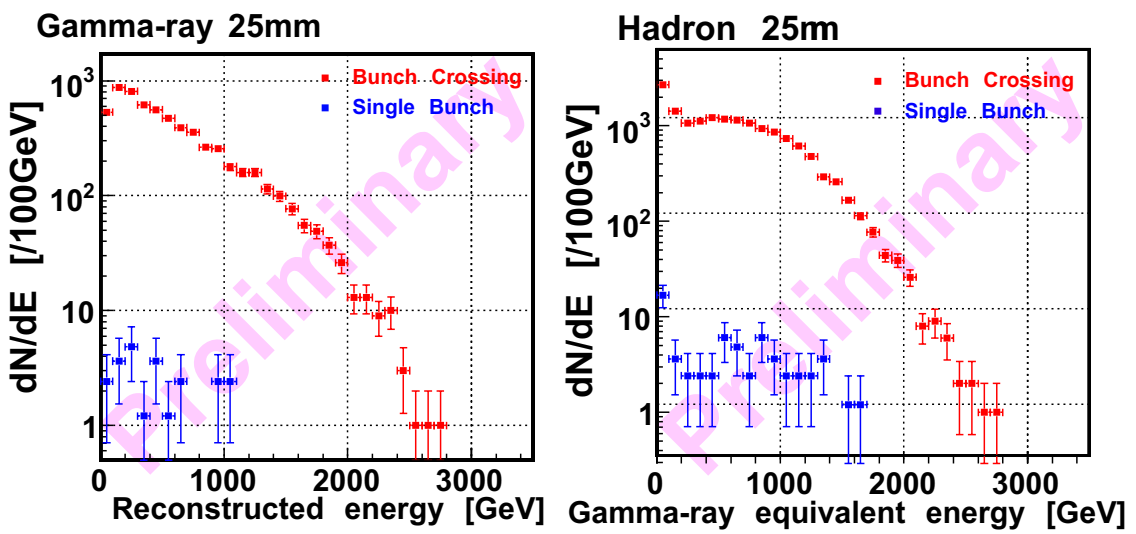

Figure 4: The energy spectra of gamma-ray like events and hadron like events measured by the Arm\#2 smaller calorimeter on March 30, 2010. The red and blue dots indicate spectra of the events at colliding and non-colliding beams at IP1, respectively.

\section{Summary}

The aim of the LHCf experiment is to measure energy and transverse momentum spectra of gamma-rays and neutral hadrons emitted in the very forward region of LHC due to calibration of hadron interaction models. In 2009, LHCf has collected about 6,500 shower events at $900 \mathrm{GeV}$ $p p$ collisions. The measured spectra of gamma-ray like events and hadron like events are in good agreement with the Monte-Carlo simulation results generated by the QGSJET2 model within error. In 2010, LHC are delivering $p p$ collisions at $\sqrt{s}=7 \mathrm{TeV}$ and LHCf are taking data with very low beam-gas background level of less than $1 \%$. LHCf will continue operation at $7 \mathrm{TeV}$ until the luminosity reaches $10^{30} \mathrm{~cm}^{-2} \mathrm{~s}^{-1}$ or the integral luminosity reaches $2 \mathrm{nb} \mathrm{b}^{-1}$.

\section{References}

[1] The LHCf Collaboration, JINST, 3 (2008) S08006

[2] S. Ostapchenko, Phys. Rev. D74 (2006) 014026. 